\title{
El talento humano y su evolución en la industria 4.0
}

Human resource management and its evolution in industry 4.0

\author{
Humberto Pedro Segarra Jaime \\ Marjorie Ordoñez Guartazaca \\ David León Ortega
}

Fecha de recepción: 03 de mayo 2020

Fecha de aceptación: 20 de junio 2020 


\title{
El talento humano y su evolución en la industria 4.0
}

\author{
Human resource management and its evolution in industry 4.0 \\ Humberto Pedro Segarra Jaime ${ }^{1}$, Marjorie Ordoñez Guartazaca², David León Ortega ${ }^{3}$
}

\section{RESUMEN}

La presente investigación tiene como objetivo mostrar las perspectivas, evolución y tendencias del talento humano en la irrupción de la industria 4.0 y sus principales características. La metodología aplicada es cualitativa con revisión bibliográfica fundamental de actualidad, bajo un diseño exploratorio y descriptivo que denota el entorno actual previo al inicio de un nuevo modelo de trabajo con el fortalecimiento y transformación de la industria a la automatización total y muestra que el talento humano debe tomar esta realidad como una oportunidad para su crecimiento y desarrollo profesional en esta nueva era de información y conocimiento.

Palabras clave: Industria 4.0, Transformación, Talento Humano

\begin{abstract}
The research proposal is to get the perspectives, evolution, and trends of human talent in the e mergence of Industry 4.0 and its main characteristics. The qualitative methodology was appli ed with a fundamental current bibliographic review, under an exploratory and descriptive desi gn that denotes the current environment before the start of a new working model with the stre ngthening and the transformation of the industry full automation and shows that human talent you must take this reality as an opportunity for your growth and professional development in this new era of information and knowledge.
\end{abstract}

Keywords: Industry 4.0, Transformation, Talent Human Management

\footnotetext{
${ }^{1}$ Magíster Ejecutivo en Dirección de Empresas. Docente en Facultad de Ciencias Administrativas de la Universidad de Guayaquil, Ecuador, Correo electrónico: humberto.segarraj@ug.edu.ec, ORCID: https://orcid.org/0000-0003-3946-952X

${ }^{2}$ Magister en Administración de Empresas. Docente en Facultad de Ciencias Administrativas de la Universidad de Guayaquil, Ecuador, Correo electrónico: marjorie.ordonezg@ug.edu.ec

${ }^{3}$ Estudiante de Universidad de Loja, Ecuador, Correo electrónico: davixo99@ gmail.com
} 


\section{INTRODUCCIÓN}

El talento humano se ha visto afectado por transformaciones y cambios llevados por los nuevos procesos productivos y las nuevas tecnologías que van desplazando a las anteriores, resultando en un fenómeno que repercute en los ambientes sociales, culturales y económicos, como lo indica Martínez (2012), y además se constituye en una oportunidad para que los profesionales de toda índole tengan nuevos ambientes de gestión en los cuales explotar sus conocimientos. En ese sentido, Moreno (2016) resalta que hay un impacto significativo en todas estas transformaciones y las mismas son derivadas por el internet, las interconexiones, las supercomputadoras, la inteligencia artificial, software más especializados y un sinnúmero de mejoras que día a día evolucionan las formas en que se percibe al mundo. De igual forma, las investigaciones afirman que "la incursión de las Tecnologías de la Información y las Comunicaciones (TIC) en el contexto organizacional ha revolucionado el pensamiento administrativo y gerencial, y esto se evidencia en varios aspectos, uno de ellos es la forma como se gestiona el talento humano" (Riascos \& Aguilera, 2011, p. 141).

El talento humano siempre estará en vigencia en cualquier revolución disruptiva que promueva una nueva forma de hacer negocios, ya que estos procesos involucran personas, y estas no son solo recursos, son talento. La dinámica del mundo empresarial actúa proponiendo cambios y mejoras no solo en los departamentos de ventas, finanzas y logística, sino en uno de los más importantes dentro de la cadena de valor, el de talento humano. La gestión humana, por tanto, ha requerido también un cambio, una transformación profunda para adaptarse, según lo indica Guasch (2018), a estos nuevos entornos, con una dosis de talento, acrecentada por un ciclo de aprendizaje continuo a lo largo de la carrera profesional de un trabajador.

Estos cambios se han producido como resultado de los movimientos de la industria desde la época que inició el comercio hasta la actualidad, donde la presencia de robots e innumerables máquinas trabajan gracias al talento de las personas que las operan. Estas operaciones se dan en las empresas dentro de una comunidad de personas, apoyada por equipos y software que facilitan su trabajo y gestión, todo esto interrelacionado con una remuneración de acuerdo a sus funciones, un buen clima laboral y la estabilidad para desarrollar su trabajo.

Las realidades empresariales que viven las compañías son cada vez más complejas. En este aspecto, Corporación industrial MDC (2016) plantea varias preguntas clave para enfrentar esta situación: ¿qué pasará en el futuro con el capital humano? ¿las nuevas tecnologías destruirán los puestos de trabajo? ¿nos quedaremos sin empleo? Estas preguntas quizás promuevan un escenario desolador; pero, contrario a esto, también podría generar más oportunidades y 
expectativas para las personas creativas y emprendedoras, dispuestas a aprender y capacitarse en habilidades digitales tomando decisiones que las máquinas, tecnología y robots, no pueden tomar.

Restrepo y Arias (2015) señalaron lo siguiente:

Dados los procesos de cambio a los que se ven sometidas las organizaciones, las prácticas de gestión humana pasan de ser un factor meramente instrumental a constituirse en un factor estratégico clave en el desarrollo de las empresas y sus trabajadores para el logro de los objetivos propuestos. (p.22)

Wright, Dunford y Snell (2001) señalan que es muy importante que los recursos humanos se convierten en una ventaja competitiva y que además es sostenible en el tiempo, por tanto invertir y fomentar el desarrollo del talento humano conlleva también a asegurar el futuro de la empresa y más aún cuando enfrenta cambios de 360 grados que se manifiestan cada vez que aparece un nuevo modelo de negocio disruptivo e innovador.

Sánchez y Herrera (2016) enfatizan que las organizaciones están en la búsqueda de formas más creativas para sus labores y, además, se están adaptando cada vez más rápido a entornos dinámicos e innovadores en donde se propende la creación de ventajes productivas y competitivas dentro de la cadena de valor de cada empresa en los distintos estilos de administración que se llevan en las compañías.

En esta época moderna, la industria 4.0 incluye la utilización amplia de la tecnología a través del Internet de las Cosas, el Big data, la ciberseguridad e incluso el cloud computing que generan una producción cada vez más próspera y eficiente, de la mano de la tecnificación y digitalización de la mano de obra. Por tanto, esta investigación se enfoca en las perspectivas que tiene la gestión del talento humano de cara a enfrentar esta revolución en el presente siglo.

\section{MARCO TEÓRICO}

\section{Capital Humano}

Chiavenato (2016) conceptualiza al capital humano como un conjunto de personas el cual se valoriza dependiendo de los talentos y competencias que generen valor para la empresa y la fortalezcan en competitividad y productividad. Este capital se consolida en 4 pilares fundamentales que son la autoridad, la información, las recompensas y las competencias, mismos que, actuando de forma sinérgica, potencializan la actuación de las personas dentro de una organización a lo largo de su desarrollo dentro de ella. Es válido señalar que "la calidad del servicio de una empresa radica en la posibilidad de sostener el talento humano en una línea temporal prolongada" (Mejía, Bravo, \& Montoya, 2013, pág. 6). 
Para Dessler (2009), el capital humano se constituye en todos los conocimientos, la educación, la capacitación, las habilidades y la pericia de los trabajadores de una compañía, dado que los mejores trabajos requieren personal preparado y capacitado. Además, el mimo autor indica que Peter Drucker predijo hace varios años, "el centro de gravedad del empleo se está moviendo rápidamente de los trabajadores manuales y de oficina a los trabajadores especializados..."

En ese sentido, Usman \& Wirawan (2021) hacen hincapié en que el capital humano se vuelve considerablemente importante porque es una fuente de innovación y renovación estratégica, y puede fomentarse a partir de la investigación aplicada, objetivo de gestión, proceso de reingeniería y mejora en el desarrollo de habilidades de la fuerza laboral. Además, dicho capital crea valores incrementales para las organizaciones diariamente a través de la motivación, el compromiso, competencia y eficacia del trabajo en equipo.

\section{Administración del Talento Humano}

Mondy (2010) define a la administración del talento humano como la coordinación de la participación de los trabajadores con la meta de cumplir los objetivos el negocio, haciendo que todos los niveles de la organicación focalicen sus esfuerzos en cubrir todas la áreas de la empresa. En otras apreciaciones, en materia de administración también "significa mantener la perspectiva de las personas que trabajan como fin, vinculada al mejoramiento humano" (Cuesta, 2010, pág. 19).

Otros autores como García, Sánchez y Zapata (2008) definen la gestión humana como: la actividad estratégica de apoyo y soporte a la dirección, compuesta por un conjunto de políticas, planes, programas y actividades, con el objeto de obtener, formar, motivar, retribuir y desarrollar al personal requerido para generar y potencializar, el management, la cultura organizacional y el capital social, donde se equilibran los diferentes intereses que convergen en la organización para lograr los objetivos de manera efectiva (p.16).

Además Cuesta (2010) incluye en esta administración un proceso estratégico el cual incluye tres dimensiones que son: recurso para la competitividad, enfoque sistemático y coherencia con la estrategia, ya que para que funcione el talento humano dentro de una empresa, sus dimensiones deben actuar de forma completa tomando en cuenta el entorno y las condiciones de mercado.

Los procesos administrativos, incluidos los de talento humano, consideran una metodología sistemática que logra maximizar los esfuerzos para la correcta administración de las personas 
en todos sus procesos de dotación de personal, reclutamiento, selección y capacitación, los cuales incluyen fases y etapas importantes para el óptimo desenvolvimiento de sus gestiones.

\section{Dotación de Personal}

El autor Mondy (2010) la denomina como un proceso que procura tener dentro de la empresa el personal idóneo en cantidad y de acuerdo al perfil en las áreas adecuadas para cumplir con éxito los objetivos de la organización. Consta de varias etapas que incluye los análisis de puestos, el cumplimiento de las normativas que intervienen y el análisis del orgánico funcional de la compañía.

En ese sentido, el análisis de puestos otorga "información acerca de lo que entraña el puesto y las características humanas que se requieren para realizar esas actividades". Por medio de estas descripciones y la especificación del puesto, "ayuda a los gerentes a decidir el tipo de personas que se reclutarán y contratarán” (Dessler, 2009, pág. 126).

Sin embargo, también es importantante destacar el hecho de que el capital humano es una cualidad dentro de un individuo, por lo que el capital social se crea entre individuos. Por ello, el entorno de trabajo que el gerente pueda crear en base a las características de cada puesto e individuo, viene a ser fundamental, pues es un recurso incrustado en las relaciones, desarrollado a través de cambios en las relaciones y reflejado en el valor de estas relaciones (Crane \& Hartwell, 2018). En consecuencia, el capital social creado a partir de la selección de personal es de particular importancia para las empresas, porque facilita la coordinación de actividades más allá del entorno físico como tal.

\section{Reclutamiento}

Dessler (2009) lo define de forma muy sencilla como la forma de encontrar o atraer candidatos que cubran las expectitivas de una vacante dentro de la empresa. El reclutamiento incluye varias etapas entre las que se pueden mencionar la selección, aplicación de técnicas, comprobación de datos y hoy en día lo puede hacer la empresa o un compañía independiente dedicada a este actividad. Para Bateman y Snell (2009) esta actividad permite tener más candidatos e incluye un reclutamiento interno y un reclutamiento externo.

El reclutamiento interno tiene grandes ventajas ya que permiten que los trabajadores tengan oportunidad de ser elegidos por sus empleadores con base a su experiencia, conocimientos y productividad dentro de la compañía. El reclutamiento externo promueve traer nuevos talentos a la compañía que pueden innovar y renovar la imagen, los procesos y las actividades empresariales, se usan fuentes como las publicaciones, el internet, los sitios de intranet de la 
compañía, las universidades, bolsas de empleo, becarios o pasantes los cuales pueden ser reclutados por una compañía externa también.

\section{Selección de Personal}

Para Chiavenato (2016), la selección "busca entre los candidatos reclutados a los más adecuados para los puestos que existen en la empresa, con la intención de mantener o aumentar la eficiencia y el desempeño del personal, así como la eficacia de la organización” (p.169). Con ello indica lo importante que significa que luego de reclutar se apruebe un proceso para la selección que cumpla los intereses de la organización, entre ellos están:

1. Solicitudes de hoja de vida

2. Entrevistas

3. Revisión de referencias

4. Revisión de antecedentes

5. Pruebas de personalidad

6. Pruebas de habilidad cognitiva

7. Pruebas de rendimiento

8. Pruebas de integridad

Todas ellas relacionadas con la comprobación de la idoneidad al puesto y se adaptan perfectamente a lo que exige la industria moderna y competitiva. Hay inclusive nuevas vertientes que ya se aplican como revisar el currículo sin foto ni nombres, esto con el objetivo de no excluir a ningún candidato y calificarlo por sus habilidades, capacidades y conocimientos.

\section{Procedimientos de Control del Talento Humano}

Soto (2017) manifiesta la importancia de los procesos de control del talento humano como imprescidibles para lograr la optimización de recursos, la eficiencia organizacional y el establecimiento de lineamientos para el mejoramiento continuo de las instituciones dirigido hacia la preparación de los involucrados formando y fortaleciendo capacidades para su labor diaria y futura.

La gestión procedimental es básica en el área donde se administra talento humano y en la dinamia actual la comunicación sigue siendo por excelencia la clave para resolver conflictos, mejorar el clima laboral, las evaluaciones de desempeño y en general todos los procesos que enlazan el talento humano en el siglo XXI. La revolución exige mayor preparación y 
calificación de los trabajadores por eso los proceso para su efecto deben ser cuidadosamente diseñados y preparados.

Las normativas laborales, leyes y otros reglamentos también deben adaptarse como en el caso del teletrabajo o freelancers, o el trabajo por horas, ya que cada vez más se están manifestando los efectos en las remuneraciones y en los procesos de contratación y pagos como la flexibilidad, la eliminación de sobres de pago y con una sencilla transferencia bancaria el cobro del sueldo en cualquier lugar del mundo prestando los servicios a distintas empresas nacionales o internacionales.

\section{Capacitación}

Parte fundamental de un proceso de cambio es la capacitación a los empleados, la cual "significa proporcionar a los empleados nuevos o antiguos las habilidades que requieren para desempeñar su trabajo" (Dessler, 2009, pág. 294). Es un proceso valioso que inicia desde la contratación del personal y se despliega durante toda la vida laboral del colaborador para que puede mantener sus habilidades y conociemientos acorde a las exigencias del puesto al que ha sido asignado.

En consecuencia, Gwan, Ki Kim, \& Mug (2020) afirman que una capacitación efectiva para lograr los objetivos propuestos puede producir mayor satisfacción y productividad en el trabajo, a la par que mejora la calidad del mismo, aumenta la motivación y compromiso, mayor moral y responsabilidad con la tarea asignada, y menos errores en el momento de ejecutar los planteamientos iniciales.

\section{Industria 4.0}

El libro "La cuarta revolución industrial" de Schwab (2016) propone el nacimiento de esta revolución que construye nuevos sistemas tecnológicos en entornos cada vez más digitales, que impulsará nuevos empleos más enriquecidos de características tecnificadas acorde a la nueva producción industrial. A saber, es un "conjunto de sistemas que combinan software, sensores, nanotecnología y una tecnología digital de comunicaciones que están construidos sobre la infraestructura de la revolución digital ya anterior".

Según el mismo Schwab (2016), la primera revolución industrial (1780-1840) se caracterizó por el uso del agua y vapor, lo que propendió a una producción mecanizada. En la segunda revolución (1870-1914) se fomentó el uso de la energía eléctrica, lo que generó la producción en masa. A partir de los nuevos avances, aparece la tercera revolución industrial (1945-1970), llena de cambios en la electrónica y las tecnologías de la información denominadas TIC. Se produce de forma automatizada en casi todos los sectores. Nace, entonces, luego de ese período, la cuarta revolución industrial (1970), hasta la actualidad. Aquí aparecen y se fusionan 
nuevas tecnologías dentro del mundo digital, físico y hasta biológico, reinando la inventiva, la disrupción y su evolución.

En consecuencia, el eje tecnológico central de la Industria 4.0 es la comunicación, intermediación y el entorno de relación (entorno 4.0) realizado a través de sistemas ciberfísicos (CPS) y los sistemas de conducción (CPPS). Dicho ambiente especializado emplea recursos humanos para efectuar procesos de pronóstico tecnológico y cambio social, actividades creativas y de resolución de problemas, y este mismo sistema garantiza su funcionalidad a través de dos factores clave: integración e interoperabilidad (Cugno et al., 2021).

Adicional a ello, Jamwal et al. (2021) mencionan que, con el desarrollo de la industrialización, la sostenibilidad de las nuevas formas de producción se ha convertido en un problema importante en el intercambio mundial. En ese sentido, al existir poca importancia y previsión de los problemas de sostenibilidad, inevitablemente se traza un camino que conduce a enormes pérdidas financieras y al debilitamiento de la industria. Por tanto, la tendencia de producción actual dentro de la Industria 4.0 ofrece nuevas tecnologías clave que están contribuyendo a la sostenibilidad de forma directa o indirecta, lo que a su vez se ha traducido en la identificación de diferentes habilidades y ha facilitado la adopción de prácticas de sostenibilidad y permanencia en el mercado.

Corporación Colombia Digital (2017) indica que esta persigue una transformación de la empresa a una computador "smart factory", como se lo denomina usualmente, y este proceso genera un tecnificación superior para realizar los controles de forma eficaz, es decir, una producción que busca la personalización a través de la automatización. En la Tabla 1 se muestran las características más representativas de esta industria.

Tabla 1

Características de la Industria 4.0

\section{Características más relevantes de la Industria 4.0}

$\begin{array}{ll}\text { Big Data } & \text { Canales (CMS, SCM, CRM, FCM, HRM, } \\ & \text { Help desk, redes sociales, IoT) } \\ \text { Almacenamiento en la nube } & \text { Impresiones 3D } \\ \text { Robótica colaborativa } & \text { Fábricas inteligentes } \\ \text { Internet de las cosas } & \text { Finanzas digitales } \\ \text { Simuladores } & \text { Inteligencia artificial } \\ \text { Personalización } & \text { Ciberseguridad }\end{array}$


Estas características se manifiestan ya en la actualidad, en el caso de la ciberseguridad, y existen profesionales que cada vez más ocupan cargos de importancia estratégica por ser conocedores del tema, mientras que los software de gestión empresarial como ERP y CRM están cada día más conectados, de forma que los datos se encuentran en la nube automáticamente, generando más personal preparado para su protección, respaldo y cuidado. En el ámbito de la inteligencia artificial (IoT) o onternet de las cosas, todo está ocurriendo a pasos agigantados, y ya desde el hogar se encuentra ejemplos de conexión entre equipos con mandos vocales que ya no son manuales.

Sin embargo, según Birkel et al. (2019), uno de los principales obstáculos a nivel global para la adopción de la Industria 4.0 está enfocado en el ámbito cultural y la aceptación por parte de recursos humanos. A saber, una cultura interna de reconocer el avance tecnológico debe sembrarse en toda la organización, para garantizar que los individuos estén preparados para adoptar nuevas tecnologías. No obstante, muchos gerentes y trabajadores siguen sin querer cambiar sus estrategias y tareas en el ámbito de la producción, y en muchos casos, existe resistencia a los nuevos métodos, por lo que resulta fundamental la intervención en los procesos de inducción de las empresas y organizaciones hacia ese nuevo modo de producción científica y tecnológica.

Si bien existen robots cada vez más inteligentes y con nuevos roles en ámbitos de producción y asistencia personalizada, aún no tienen cualidades innatas de los seres humanos como la creatividad, el sentido común, la conciencia y saber recrearse junto con otros individuos. Esas cualidades hacen que el humano se sienta único y que no pueda reemplazarse con un robot con quizás inteligencia superior, pero que aún no tiene la capacidad de tomar decisiones.

Por otro lado, Schwab, Gold \& Reiner (2019) observan que es fundamental que exista un requisito de identificación de facilitadores clave para las organizaciones que están dentro de la industria 4.0. A saber, hay tres dimensiones para lograr la sostenibilidad de esta industria: social, económica y medioambiental. Las empresas y organizaciones que logren posicionarse en estas tres áreas, podrán establecer patrones que les permitan mejorar sus procesos, optimizar recursos productivos, digitalizar cadenas de suministros, establecer métricas de rendimiento para sus operaciones, entre otras.

\section{Tendencias del Talento Humano}

Deloitte (2016) identifica en su estudio internacional 10 principales tendencias que se enfocan principalmente en las personas y sus capacidades para desarrollar aprendizaje, nuevas competencias, cultura, compromiso, entre otras. Estos factores promueven un mejor trabajo en equipo, un mayor desarrollo en la organización y una gestión más eficiente. La Figura 1 
muestra las tendencias en su orden de importancia de acuerdo a los resultados de la investigación.

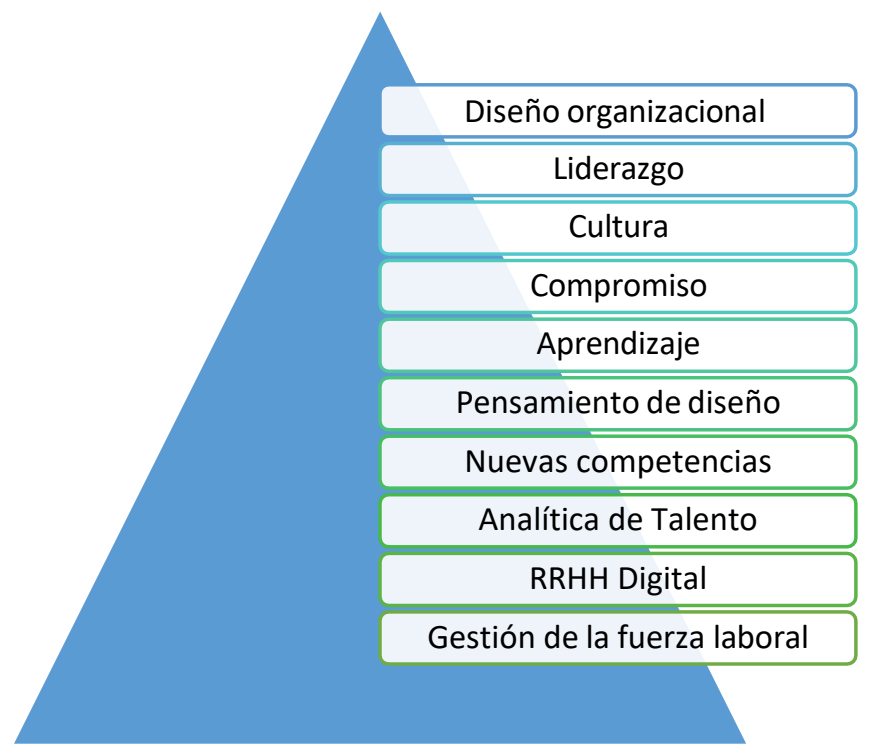

Figura 1. Tendencias del Talento Humano

En la conversión de la economía digital y la industria 4.0 se manejan nuevas variables que, de acuerdo a EC BRANDS (2017), deben ser conocidas, ya que muchas empresas ya están cambiando sus obsoletos modelos por nuevas formas de negocio. Estos variables son:
1. El consumidor es digital
2. El mercado también es digital
3. I+D: obligatorio, no opcional
4. Disrupción Tecnológica
5. Incertidumbre del mercado
6. Vigilancia en los sectores

\section{MÉTODOS Y MATERIALES}

Según Hueso y Cascant (2012), la metodología se constituye en la estrategia para responder a las interrogantes de investigación que se plantean en el transcurso de la misma. Se usará un enfoque cualitativo y fue necesario la recolección de datos para su análisis. Se utilizó el método deductivo en el análisis de los estudios, teorías y los artículos a nivel mundial sobre el tema de la Industria 4.0 y su afectación al talento humano organizacional. La investigación es exploratoria y descriptiva ya que confiere importancia al estudio del entorno actual y sus implicaciones al tema. 
Asimismo, es importante tener en cuenta la unidad de análisis, que en este caso es la Industria 4.0. En ese sentido, es fundamental realizar una definición apropiada de la unidad de análisis, lo que debe contribuir a delimitar las fronteras del estudio. Por tanto, Villarreal \& Landeta (2010) exponen que la definición de la unidad de análisis, y con ello del tipo de metodología, se relaciona con la forma en que ha sido definida la cuestión de investigación inicial. Cabe destacar que cuando existen estudios previos, estos establecen algún criterio en la elección con el fin de poder comparar resultados y obtener conclusiones robustas.

\section{RESULTADOS Y DISCUSIÓN}

La investigación muestra los siguientes hallazgos de relevancia para el Talento Humano en aras de volverse más competitivo y productivo en los nuevos escenarios económicos y sociales de esta revolución. Así, de acuerdo a Cobián (2017), los empleos que tendrán más demanda durante la consolidación de la Cuarta Revolución Industrial son:

- $\quad$ Analista de data.

- $\quad$ Especialista en representante de ventas.

- $\quad$ Especialista en el desarrollo operacional de una empresa.

- Ingeniería bioquímica.

- Ingeniería en nanotecnología y robótica.

- $\quad$ Expertos en sistemas de información.

- $\quad$ Operadores de drones.

- $\quad$ Expertos en la generación de millenials.

- $\quad$ Gerente de sustentabilidad.

- $\quad$ Especialista en computarización de la nube (Cloud).

- Ingeniería automotriz automática (sin conductor).

Asimismo, otro factor de igual o más trascendencia, son las características que se fortalecerán en el Talento Humano para acrecentar sus posibilidades de sostener la Industria 4.0. Entre ellas, en relación a un análisis de World Economic Forum (2018), se enlistan en la Figura 2: 


\section{in 2020}

1. Complex Problem Solving

2. Critical Thinking

3. Creativity

4. People Management

5. Coordinating with Others

6. Emotional Intelligence

7. Judgment and Decision Making

8. Service Orientation

9. Negotiation

10. Cognitive Flexibility

\section{in 2015}

Complex Problem Solving

Coordinating with Others

People Management

Critical Thinking

Negotiation

Quality Control

Service Orientation

Judgment and Decision Making

Active Listening

10. Creativity
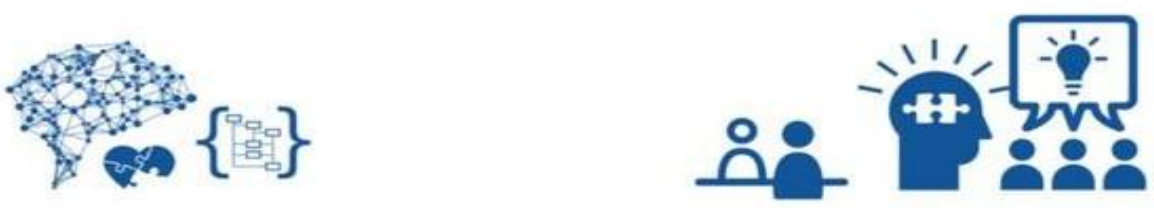

Source: Future of Jobs Report, World Economic Forum

Figura 2. Características del Talento Humano en el futuro.

Estas 10 características incluyen: i) resolución de problemas complejos, puesto que la automatización lleva a razonamientos más complejos, ii) el pensamiento crítico, este factor es fundamental para la toma de decisiones, iii) creatividad, dado que los negocios de hoy no prosperan si no existe la innovación y creación constante, iv) manejo de personas, pues la gestión del talento es parte de estos cambios, v) coordinación con otros, es vital saber trabajar en equipo y colaborativamente, vi) inteligencia emocional, básico para el ciudadano del siglo XXI, vii) juzgar y tomar decisiones, una cualidad potenciada en esta nueva era, viii) orientación de servicios enfocada en el cliente, ix) negociación en todos los sentidos y aspectos empresariales $\mathrm{y}$, por último, $\mathrm{x}$ ) flexibilidad cognitiva, que se vuelve esencial para cambiar conceptos, es decir, crear.

A saber, es fundamental que actúen en forma coordinada 4 factores básicos que promueve el establecimiento de la industria 4.0 y que deben robustecer a las organizaciones y al personal que forma parte de ellas. La Figura 3 muestra la adaptación de la propuesta de Schwab (2016) de este nuevo modelo que representa el establecimiento de esta revolución. 


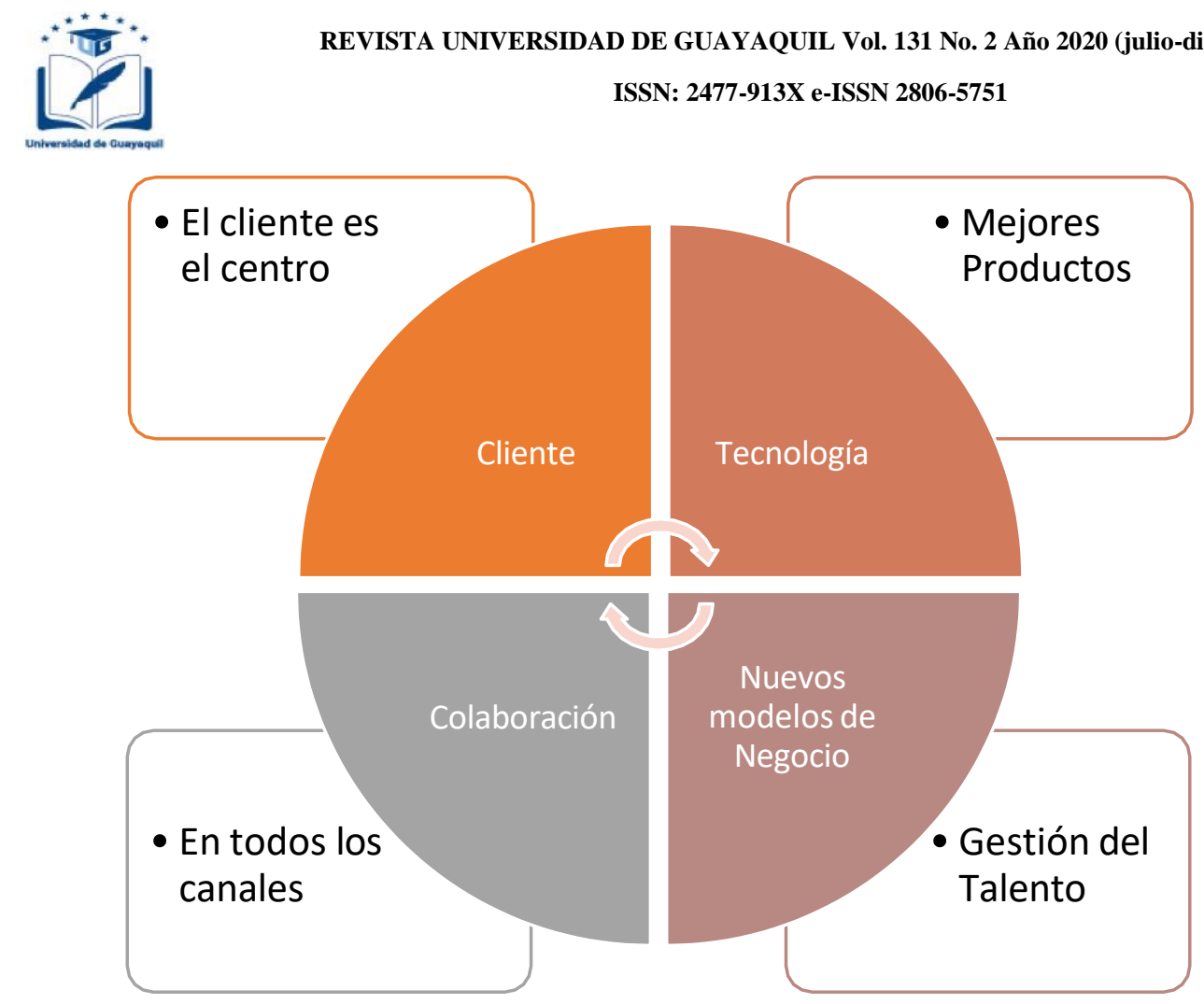

Figura 3. Factores fundamentales para enfrentar a la Industria 4.0

Las nuevas profesiones que genera la industria con énfasis en la tecnología, las nuevas características de los profesionales para cubrir esos nuevos puestos y los factores para llevar a éxito esta revolución, son los principales hallazgos de la investigación, mismos que generan información de relevancia para la gestión del talento humano que debe contribuir al cambio, con nuevos enfoques y propuestas para su innovación y crecimiento.

El informe de Deloitte (2016) sobre las tendencias globales en Capital Humano generan una importante relación con algunos factores organizacionales que forman, finalmente, la conjunción de los esfuerzos por un sano ambiente laboral, y con el advenimiento de esta nueva tendencia, las máquinas, robots, inteligencia artificial, internet de las cosas y otros, serán parte de la vida diaria del trabajador, donde aparece un liderazgo inspirador que se manifiesta por parte de las autoridades, así como también metas, visión, compromiso, reconocimientos laborales, riesgos y remuneración competitiva, que generen confianza para que el trabajador enfrente el reto de forma realista y optimista, conociendo sus potencialidades y con apoyo de sus directivos logrará sobrepasar las expectativas que genera estar en esta nueva industria. La Figura 4 detalla los aspectos más relevantes de estos vínculos laborales de cultura y compromiso. 


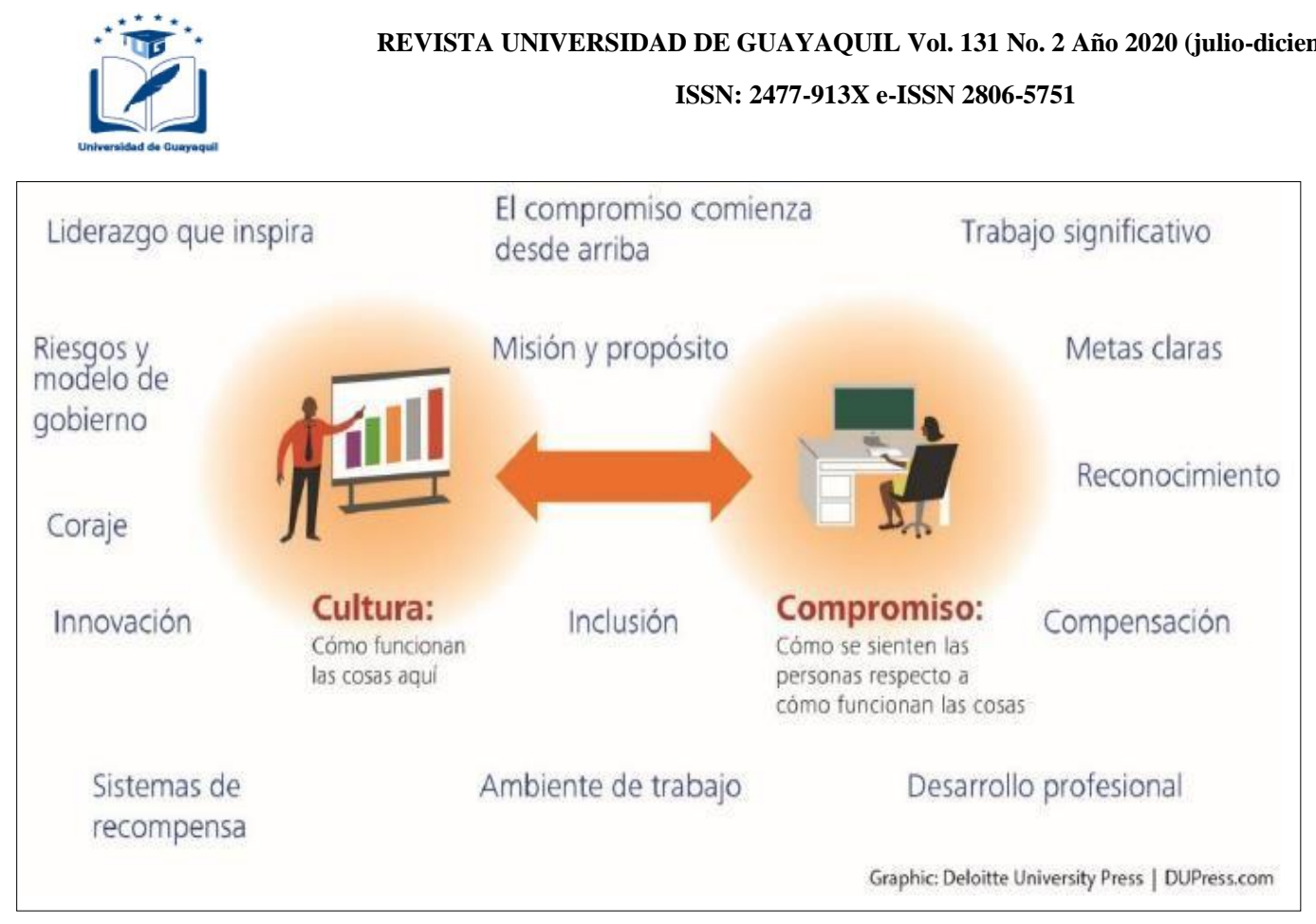

Figura 4. Cultura y Compromiso Laboral

Fuente: Informe de Tendencias Globales del Capital Humano, Deloitte (2016)

Finalmente, resulta fundamental enlazar la información presentada por Schwab, Gold \& Reiner (2019) con los resultados obtenidos. A saber, las organizaciones y empresas que han enfocado sus esfuerzos en posicionarse en las tres dimensiones de esta industria (social, económica y medioambiental), han logrado seguir una dirección en que, por ejemplo, la cadena de suministro y los facilitadores de la dimensión ambiental ejercen una fuerte influencia sobre la adopción de prácticas de sostenibilidad en el largo plazo, lo que les ha permitido automatizar procesos y optimizar recursos, generando un panorama estable y próspero.

\section{CONCLUSIONES}

Para las economías latinoamericanas se plantean grandes desafíos en la industria 4.0, puesto que se la efectivización de la misma generará productividad en todos los sectores, haciendo más competitivas a las empresas y, a través de este medio, más productivas a las personas que la forman. La cadena de valor empresarial tendrá gran presencia debido a la transformación que los perfiles laborales requerirán para adaptarse y entrar en el cambio industrial.

La independencia total de la mano de obra será lo que depare el futuro y los trabajadores deben estar preparados para esas nuevas realidades y entornos laborales, en donde predominará la especialización, la polivalencia y la integración de analistas, que serán lo más importante en la organización, a pesar de la completa automatización.

Los retos y oportunidades son extensas, principalmente para las economías en desarrollo, como nuestros países. Por ello, es importante la preparación y el continuo aprendizaje, ya que las capacidades digitales y de conocimiento son las que se pondrán a prueba para el futuro talento 
humano, el cual buscar aumentar sus ingresos y mejorar sus niveles de vida como un objetivo profesional y personal.

Es vital que las nuevas empresas y organizaciones empiecen implementando procesos de postulación y selección de personal, en los que predomine la capacitación en habilidades tecnológicas, automatización y optimización de recursos, ya que el mercado global se irá ajustando a los cambios en los patrones de producción y consumo, y el sector industrial tendrá que avocarse hacia una adaptación organizada y resiliente si es que desean permanecer en el entorno productivo.

\section{REFERENCIAS}

Bateman, T., \& Snell, S. (2009). Administración Liderazgo y colaboración en un mundo competitivo (Octava ed.). México: McGraw-Hill.

Birkel , H., Veile, J., Müller , J., Hartmann , E., \& Voigt , K.-I. (2019). Development of a Risk Framework for Industry 4.0 in the Context of Sustainability for Established Manufacturers. Sustainability, 384. doi:https://doi.org/10.3390/su11020384

Chiavenato, I. (2016). Administración de Recursos Humanos (Octava ed.). México: McGrawHill.

Cobián, C. (15 de Marzo de 2017). IMPACTO DE LA CUARTA REVOLUCIÓN INDUSTRIAL EN. Obtenido de https://goo.gl/pkemMu

Corporación Colombia Digital. (3 de Junio de 2017). Industria 4.0, la cuarta revolución industrial impacta la fabricación y manufactura. Recuperado el 7 de Febrero de 2018, de https://goo.gl/GM36cW

Corporación industrial MDC. (14 de Diciembre de 2016). Capital humano en la industria 4.0: ¿nos quedaremos sin trabajo? p. 4. Obtenido de https://mdc.org.co/2016/12/14/capitalhumanolaindustria-4-0-nos-quedaremos-sin-trabajo/

Crane, B., \& Hartwell, C. (2018). Global talent management: A life cycle view of the interaction between human and social capital. Journal of World Business. doi:https://doi.org/10.1016/j.jwb.2018.11.002

Cuesta, A. (2010). Gestión del Talento Humano y del Conocimiento (Segunda ed.). Meéxico: ECOE Ediciones. 
Cugno, M., Castagnoli , R., \& Büchi, G. (2021). Openness to Industry 4.0 and performance: The impact of barriers and incentives. Technological Forecasting \& Social Change. doi:https://doi.org/10.1016/j.techfore.2021.120756

Deloitte. (2016). Tendencias Globales del Capital Humano 2016. New York: Dupress.

Dessler, G. (2009). Administración de recursos humanos (Décimo Primera Edición ed.). México: Pearson Educación.

EC BRANDS. (20 de Diciembre de 2017). Llega la cuarta revolución industrial: así cambiarán las empresas de automoción. El Confidencial, p. 5.

García, M., Sánchez, K., \& Zapata, A. ((compiladores) 2008). Perspectivas Teóricas para el estudio de la Gestión Humana. Una relación con el capital social, la cultura organizacional y el management. Cali, Colombia: Programa Editorial Universidad del Valle.

Gwan, M., Ki Kim, Y., \& Mug, D. (2020). Relationship Between Job Training and Subjective Well-being In Accordance With Work Creativity, Task Variety, and Occupation. Safety and Health at Work, 466-478. doi:https://doi.org/10.1016/j.shaw.2020.08.006

Guasch, J. (7 de Febrero de 2018). La industria 4.0 requiere dosis de talento y de innovación tecnológica". (Eurecat, Entrevistador) Obtenido de https://eurecat.org/es/la-industria4-0-requiere-dosis-de-talento-y-de-innovacion-tecnologica/

Hernández, R., Fernández, C., \& Baptista, P. (2014). Metodología de la Investigación. México D.F.: Mc Graw Hill Education.

Hueso, A., \& Cascant, M. (2012). Metología y Técnicas Cuantitativas de Investigación (Primera ed.). Madrid: Editorial Universitat Politécnica de València.

Jamwal, A., Agrawal, R., Sharma, M., Kumar, V., \& Kumar, S. (2021). Developing A sustainability framework for Industry 4.0. Procedia, 430-435. doi:10.1016/j.procir.2021.01.129

Martínez, R. 2. (2012). El teletrabajo como tendencia del mercado laboral. Retos, II(4), 143156. doi: http://dx.doi.org/10.17163/ret.n4.2012.01 
Mejía, A., Bravo, M., \& Montoya, A. (2013). El factor del talento humano en las organizaciones. Ingeniería Industrial, XXXIV(1), 2-11. Obtenido de http://scielo.sld.cu/pdf/rii/v34n1/rii02113.pdf

Mondy, R. (2010). Administración de Recursos Humanos (Décimo Primera ed.). México: Pearson Education.

Moreno, M. (2016). Opciones de mejora cognitiva no convencional como respuesta al desempleo estructural en el contexto tecnológico de la cuarta revolución industrial. Gazeta de Antropología, 32(2), 1-19. Obtenido de http://hdl.handle.net/10481/43309

Restrepo, F., \& Arias, F. (2015). Las prácticas de gestión del talento humano en empresas agropecuarias del sector bananero en Colombia. Journal of Agriculture and Animal Sciences, $I V(2), 20-32$.

Riascos, S., \& Aguilera, A. (2011). Herramientas TIC como apoyo a la gestión del talento humano. Cuadernos de Administración - Universidad del Valle, 27(46), 141-154.

Sánchez, S., \& Herrera, M. (2016). Los recursos humanos bajo el enfoque de la teoría de los recursos y capacidades. xxiv(2), 133-146. doi:ttp://dx.doi.org/10.18359/rfce.2216

Schwab, K. (2016). La cuarta revolución industrial. Madrid: Penguin Randon House.

Schwab, L., Gold, S., \& Reiner, G. (2019). Exploring financial sustainability of SMEs during periods of production growth: A simulation study. International Journal of Production Economics, 212, 8-18.

Slinger, R. (2015). Modelo de gestión de talento humano basado en la provisión para la contratación de personal ejecutivo y profesional en la Fundación Centro Nacional de Medicina Popular Tradicional FCNMPT Estelí 2014-2015 (tesis de posgrado). Estelí: Universidad Nacional Autónoma de Nicaragua.

Soto, G. (2017). Propuesta de procedimientos de control interno para el área de Talento Humano. Revista Publicando, 4(13 (2)), 14-32.

Usman, A., \& Wirawan , H. (2021). The effect of human capital and physical capital on regional financial condition: the moderating effect of management control system. Heliyon, vol. 7. doi:https://doi.org/10.1016/j.heliyon.2021.e06945 
Villarreal, O., \& Landeta, J. (2010). El estudio de casos como metodología de investigación científica en dirección y economía de la empresa. Una aplicación a la internacionalización. Investigaciones Europeas de Dirección y Economía de la Empresa, vol. 16, 31-52.

World Economic Forum. (12 de Enero de 2018). The Future of job. Obtenido de http://reports.weforum.org/future-of-jobs-2016/

Wright, P., Dunford, B., \& Snell, S. (2001). Human resources and the resource based view for the firm. Journal of Management(27), 701-721. doi:doi:10.1177/014920630102700607 